\title{
Research of Compound Control for DC Motor System Based on Global Sliding Mode Disturbance Observer
}

\author{
He Zhang, Liang Ge, Mingjiang Shi, and Qing Yang \\ Southwest Petroleum University, Chengdu 610500, China \\ Correspondence should be addressed to He Zhang; zhanghe_spu_edu@163.com
}

Received 13 December 2013; Revised 10 February 2014; Accepted 22 February 2014; Published 24 March 2014

Academic Editor: Mingcong Deng

Copyright ( $2014 \mathrm{He}$ Zhang et al. This is an open access article distributed under the Creative Commons Attribution License, which permits unrestricted use, distribution, and reproduction in any medium, provided the original work is properly cited.

\begin{abstract}
Aiming at the problems of modeling errors, parameter variations, and load moment disturbances in DC motor control system, one global sliding mode disturbance observer (GSMDO) is proposed based on the global sliding mode (GSM) control theory. The output of GSMDO is used as the disturbance compensation in control system, which can improve the robust performance of DC motor control system. Based on the designed GSMDO in inner loop, one compound controller, composed of a feedback controller and a feedforward controller, is proposed in order to realize the position tracking of DC motor system. The gains of feedback controller are obtained by means of linear quadratic regulator (LQR) optimal control theory. Simulation results present that the proposed control scheme possesses better tracking properties and stronger robustness against modeling errors, parameter variations, and friction moment disturbances. Moreover, its structure is simple; therefore it is easy to be implemented in engineering.
\end{abstract}

\section{Introduction}

Since the novel magnetic material-rare earth alloy being successfully developed and applied to brushless DC motor, the response speed and the torque of DC motor have been improved. Moreover, the power/mass ratio has been greatly increased. Therefore, DC motor has been widely used in various servo devices including flight simulator [1], optoelectronic tracking platform [2], and missile electromechanical actuator [3]. Meanwhile, the requirement of the process environment on the performances of DC motor system is increasing. However, because of many uncertainties existing in the motor servo system, including modeling errors, parameter perturbations, and torque disturbances, the further improvement of its performance is hindered. The traditional PID control is no longer suitable for the complicated working environment, so the research of new servo control technology is very necessary for DC motor system.

The closed-loop robust control method, based on disturbance observer, has a strong inhibitory effect against a variety of external disturbances and small parameter variations, and then it can significantly improve the control performance of system. Therefore, it has become a basic control method for high-precision servo system and has been widely used in various types of servo systems [4-6]. Because sliding mode variable structure control (SMVSC) is a kind of nonlinear control with strong robustness against parameter perturbations and external disturbances [7-10], we can introduce SMVSC to construct the disturbance observer, namely, sliding mode disturbance observer (SMDO), which can ensure more accurate estimation on system disturbances [11, 12]. Moreover, the global sliding mode control (GSMC), which can make system trajectory initially set on the sliding surface by constructing nonlinear switching function and then eliminate the initial reaching motion, has aroused researchers' interest $[13,14]$. Therefore, this paper introduces the GSMC theory to construct the disturbance observer, namely, global sliding mode disturbance observer (GSMDO). The GSMDO is used as the inner-loop control of DC motor system in order to improve the robust performance.

For the general feedback closed-loop control system, it can transform into a compound control system by adding one or more compensation networks (i.e., feedforward controller). The compound control system possesses high control accuracy, simple structure, and good reliability. Moreover, the compound control strategy solves the contradiction between reducing errors and maintaining stability in general feedback 


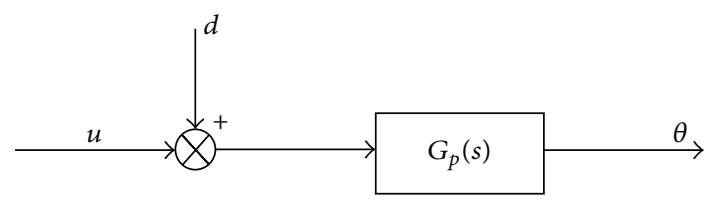

FIGURE 1: Structure diagram of open-loop DC motor system.

control system. Therefore, when the compound control technology is applied to servo system, high speed and high accuracy of position tracking can be achieved [15].

In order to guarantee the engineering practicability (related to simple design) and the method effectiveness (related to small tracking error and strong robustness against varying disturbances), we intend to explore a novel servo control method for DC motor system in this paper. As mentioned before, the control scheme, which is mainly formed by an inner-loop disturbance observer and an outerloop compound controller, can not only solve the impacts of external disturbances and parameter perturbations in system, but also improve the accuracy of position tracking. Therefore, introducing this compound control to DC motor system is the main starting point of this paper.

The rest parts of this paper are shown as follows. Section 2 provides the mathematical modeling of DC motor servo system. In Section 3, based on the idea of double loop control, the GSMDO in inner loop and the compound controller in outer loop are designed, respectively. In Section 4, the performance advantages of the proposed control scheme compared to the traditional control schemes are verified through computer simulation. In addition, the relevant conclusions and future works will be given in Section 5 .

\section{Problem Description}

Considering the equivalent disturbances, which include modeling errors, parameter perturbations, and torque disturbances, the transfer function of DC motor system can be expressed as follows [15]:

$$
\theta(s)=G_{p}(s)[U(s)+D(s)], \quad G_{p}(s)=\frac{1}{J_{n} s^{2}+B_{n} s},
$$

where $J_{n}$ represents the nominal parameter with respect to equivalent moment of inertia and $B_{n}$ represents the nominal parameter with respect to equivalent damping ratio. $u$ and $d$ represent the control input and the equivalent disturbances, $\theta$ represents the angle output of motor, and $G_{p}(s)$ represents the open-loop transfer function.

Thus, the open-loop architecture of the DC motor system is shown in Figure 1.

To facilitate the next design and analysis, (1) is written in the following form of state space:

$$
\begin{gathered}
\dot{\theta}=\omega, \\
\dot{\omega}=-\frac{B_{n}}{J_{n}} \omega+\frac{1}{J_{n}}(u+d),
\end{gathered}
$$

where $\omega$ represents the angular speed of motor. Then, we can rewrite (2) in the general form of state space

$$
\dot{\mathbf{x}}=\mathbf{A}_{n} \mathbf{x}+\mathbf{b}_{n}(u+d),
$$

where

$$
\mathbf{A}_{n}=\left[\begin{array}{cc}
0 & 1 \\
0 & -\frac{B_{n}}{J_{n}}
\end{array}\right], \quad \mathbf{b}_{n}=\left[\begin{array}{c}
0 \\
\frac{1}{J_{n}}
\end{array}\right], \quad \mathbf{x}=\left[\begin{array}{c}
\theta \\
\omega
\end{array}\right] .
$$

Thus, the mathematical models (1)-(3) lay the theoretical foundation for the design of servo controller, which will be given in the next section.

\section{Servo Control Scheme Design}

3.1. GSMDO Design. From Figure 1, if the equivalent disturbance $d$ of DC motor system is estimated by some estimation methods, the same amount of compensation to control input $u$ will effectively inhibit the impact of equivalent disturbances on the control system. Based on this control thought, we will design a GSMDO for DC motor system according to model (2).

We introduce a variable $\widehat{\omega}$, which is constructed as follows:

$$
\dot{\widehat{\omega}}=-\frac{B_{n}}{J_{n}} \widehat{\omega}+\frac{1}{J_{n}} u+\frac{1}{J_{n}} \eta
$$

where $\eta$ is the input of GSMDO.

Then, we acquire the estimation error of angular speed

$$
\omega_{e}=\widehat{\omega}-\omega
$$

From (1), (5), and (6), we can obtain

$$
\dot{\omega}_{e}=a \omega_{e}+b(\eta-d)
$$

where $a=-B_{n} / J_{n}, b=1 / J_{n}$.

The global switching function is chosen as

$$
s=c\left(\omega_{e}-\omega_{e 0}\right)-c(a-b k) \int_{0}^{t} \omega_{e}(\tau) d \tau, \quad c>0, k>0,
$$

where $\omega_{e 0}$ represents the initial estimation error of angular speed. According to $s(0)=0$, system trajectory is initially set on the designed sliding surface, which means that the initial reaching phase has been eliminated.

Assumption 1. Assume the equivalent disturbance $d$ is bounded, and $|d| \leq \rho$.

Theorem 2. Choosing the switching function (8), we can design the input of GSMDO as

$$
\eta=-k \omega_{e}-q \operatorname{sgn}(s)
$$

and then design the estimation law for equivalent disturbances as

$$
\widehat{d}=-J_{n} \dot{\omega}_{e}-\left(B_{n}+k\right) \omega_{e}-q \operatorname{sgn}(s) .
$$

If $q>\rho$ is satisfied, the estimation error of angular speed will be close to zero exponentially, and then the design effectiveness and the robustness of GSMDO will be guaranteed. 
Proof. With the differential operation on (8), we can obtain

$$
\dot{s}=c \dot{\omega}_{e}-c(a-b k) \omega_{e}
$$

Substituting (7) and (9) into (11), we can obtain

$$
\begin{aligned}
\dot{s} & =c\left[a \omega_{e}+b(\eta-d)\right]-c(a-b k) \omega_{e} \\
& =c\left\{a \omega_{e}+b\left[-k \omega_{e}-q \operatorname{sgn}(s)-d\right]\right\}-c(a-b k) \omega_{e} \\
& =-c b[q \operatorname{sgn}(s)+d] .
\end{aligned}
$$

Because $c b>0$, we can get (13) according to (12). Consider the following:

$$
\begin{aligned}
s \dot{s} & =-c b s[q \operatorname{sgn}(s)+d] \\
& =-c b|s|[q+d \operatorname{sgn}(s)] \leq-c b|s|(q-\rho) .
\end{aligned}
$$

Thus, if the condition $q>\rho$ is satisfied, $s \dot{s} \leq 0$ will be established. Moreover, when and only when $s=0$, $s \dot{s}=0$ holds. Therefore, the reachability condition for sliding mode is guaranteed.

When the system trajectory is moving on sliding mode surface, we can obtain (14) according to $\dot{s}=0$. Consider the following:

$$
\dot{s}=c b(\eta-d)+c b k \omega_{e}=0 .
$$

From (14), the equivalent control in sliding mode is given by

$$
\eta_{e q}=-k \omega_{e}+d
$$
obtain

Taking the equivalent control (15) into system (7), we can

$$
\dot{\omega}_{e}=(a-b k) \omega_{e} .
$$

Because $a-b k<0$, the process of sliding mode can be described as follows: $\omega_{e}$ converges to zero exponentially. Then, the input of GSMDO is bounded in the control period, which guarantees the design effectiveness of the proposed GSMDO.

In this case, according to (7), (9), and (10), we can obtain that the output of GSMDO $\widehat{d}$ will equal the actual equivalent disturbances $d$, which guarantees the robust property of control system based on the inner-loop GSMDO. Therefore, the proof of Theorem 2 has been completed.

Because (10) will be introduced as a disturbance compensation term to the control input, it can be further improved in order to avoid the high-frequency chattering, which results from the signum term. Then, the output of GSMDO is rewritten as follows:

$$
\widehat{d}=-J_{n} \dot{\omega}_{e}-\left(B_{n}+k\right) \omega_{e}-q \operatorname{sat}(s),
$$

where

$$
\operatorname{sat}(s)=\left\{\begin{array}{ll}
\operatorname{sgn}(s) & |s|>\phi \\
\frac{s}{\phi} & |s| \leq \phi,
\end{array} \quad \phi>0 .\right.
$$

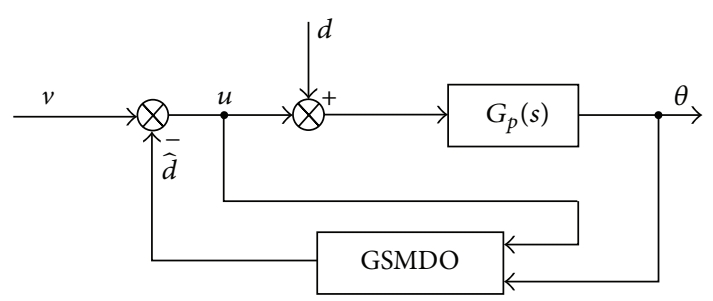

FIGURE 2: Diagram of inner-loop system structure based on GSMDO.

Thus, the inner-loop system structure based on GSMDO is shown in Figure 2.

In Figure 2, $v$ is the output of outer-loop controller, because GSMDO can only guarantee the robust performance but not the position tracking function of DC motor system. Therefore, it is necessary to design an outer-loop controller, which will be given in the next subsection.

3.2. Compound Controller Design. Owing to the introduction of disturbance compensation from GSMDO, system (3) with GSMDO can be approximately written in the following form:

$$
\dot{\mathbf{x}}=\mathbf{A}_{n} \mathbf{x}+\mathbf{b}_{n} v
$$

where $\mathbf{x}, \mathbf{A}_{n}$, and $\mathbf{b}_{n}$ have been shown below (3).

For system (19), we can design an outer-loop controller in the compound form, namely, the combination of a feedback controller and a feedforward controller. Then, the structure of the compound controller is illustrated in Figure 3.

In Figure 3, $\theta_{d}$ denotes the position tracking command of DC motor system, and $s$ denotes a differential operator. As it can be seen from Figure 3, the compound controller contains four control parameters. Subsequently, how to select the values of them to ensure the better performance of the outer-loop controller is very critical.

We introduce the state error vector $\mathbf{x}_{e}$ :

$$
\mathbf{x}_{e}=\mathbf{x}_{d}-\mathbf{x}, \quad \mathbf{x}_{d}=\left[\begin{array}{ll}
\theta_{d} & \dot{\theta}_{d}
\end{array}\right]^{T},
$$

where $\dot{\theta}_{d}$ denotes the angular velocity command of DC motor system.

With the variable substitution for system (19), we can get its equivalent system as follows:

$$
\dot{\mathbf{x}}_{e}=\mathbf{A}_{n} \mathbf{x}_{e}+\mathbf{b}_{n} v_{1}
$$

where

$$
v_{1}=-v+J_{n} \ddot{\theta}_{d}+B_{n} \dot{\theta}_{d}
$$

Moreover, $\ddot{\theta}_{d}$ denotes the angular acceleration command of DC motor system.

As a result, the state tracking problem of system (19) will be converted into the state regulating problem of system (21), which can simplify the design process. For system (21), based on LQR optimal control theory [16-18], we can design 


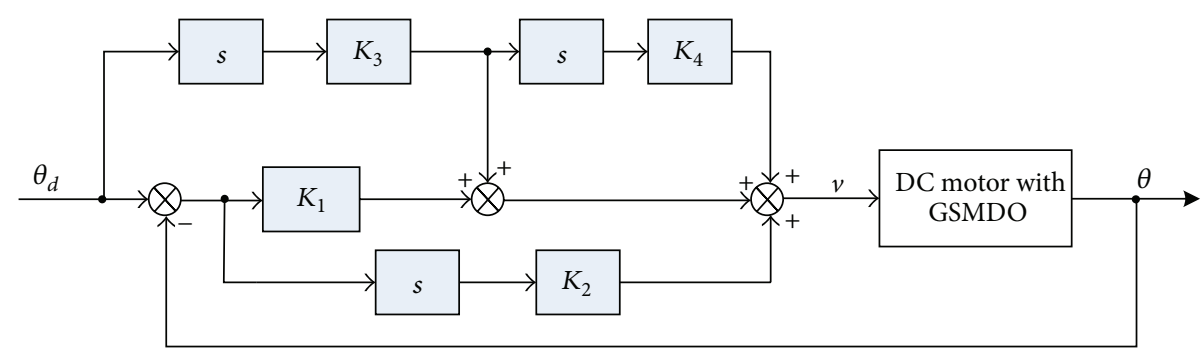

FIGURE 3: Diagram of outer-loop system structure based on GSMDO.

the feedback control law, which minimizes the following quadratic performance index:

$$
J=\frac{1}{2} \int_{0}^{\infty}\left(\mathbf{x}_{e}^{T} \mathbf{Q} \mathbf{x}_{e}+R v_{1}^{2}\right) d t,
$$

where $\mathbf{Q}$ is generally selected as a positive definite symmetric matrix and $R$ is a positive number.

In this case, the optimal feedback control law can be expressed as

$$
v_{1}^{*}=-\mathbf{k}^{*} \mathbf{x}_{e}, \quad \mathbf{k}^{*}=R^{-1} \mathbf{b}_{n}^{T} \mathbf{P}=\left[\begin{array}{ll}
k_{1}^{*} & k_{2}^{*}
\end{array}\right],
$$

where $\mathbf{k}^{*}$ represents the optimal feedback vector and $\mathbf{P}$ is a positive definite symmetric matrix and it satisfies the following Riccati equation:

$$
-\mathbf{P} \mathbf{A}_{n}-\mathbf{A}_{n}^{T} \mathbf{P}+\mathbf{P} \mathbf{b}_{n} R^{-1} \mathbf{b}_{n}^{T} \mathbf{P}-\mathbf{Q}=\mathbf{0} .
$$

Theorem 3. Based on the designed inner-loop GSMDO, the outer-loop compound controller can be designed as

$$
v=\mathbf{k}^{*} \mathbf{x}_{e}+J_{n} \ddot{\theta}_{d}+B_{n} \dot{\theta}_{d} .
$$

Then, the close-loop control system shown in Figure 3 is stable and its state vector satisfies $\mathbf{x} \rightarrow \mathbf{x}_{d}$.

Proof. Based on the designed inner-loop GSMDO, the control law (26) is designed for system (19). Correspondingly, the control law (27) can be employed to the equivalent system (21), and it is expressed by

$$
v_{1}=-v+J_{n} \ddot{\theta}_{d}+B_{n} \dot{\theta}_{d}=-\mathbf{k}^{*} \mathbf{x}_{e} .
$$

Choosing the Lyapunov function:

$$
V=\mathbf{x}_{e}^{T} \mathbf{P} \mathbf{x}_{e}
$$

with the derivative operation on (28), we can get

$$
\dot{V}=\dot{\mathbf{x}}_{e}^{T} \mathbf{P} \mathbf{x}_{e}+\mathbf{x}_{e}^{T} \mathbf{P} \dot{\mathbf{x}}_{e} .
$$

Substituting (21), (24), and (27) into (29), we can further get

$$
\begin{aligned}
\dot{V}= & \left(\mathbf{A}_{n} \mathbf{x}_{e}+\mathbf{b}_{n} v_{1}\right)^{T} \mathbf{P} \mathbf{x}_{e}+\mathbf{x}_{e}^{T} \mathbf{P}\left(\mathbf{A}_{n} \mathbf{x}_{e}+\mathbf{b}_{n} v_{1}\right) \\
= & \left(\mathbf{A}_{n} \mathbf{x}_{e}-\mathbf{b}_{n} R^{-1} \mathbf{b}_{n}^{T} \mathbf{P} \mathbf{x}_{e}\right)^{T} \mathbf{P} \mathbf{x}_{e} \\
& +\mathbf{x}_{e}^{T} \mathbf{P}\left(\mathbf{A}_{n} \mathbf{x}_{e}-\mathbf{b}_{n} R^{-1} \mathbf{b}_{n}^{T} \mathbf{P} \mathbf{x}_{e}\right) \\
= & \mathbf{x}_{e}^{T}\left(\mathbf{A}_{n}^{T} \mathbf{P}+\mathbf{P} \mathbf{A}_{n}-\mathbf{P} \mathbf{b}_{n} R^{-1} \mathbf{b}_{n}^{T} \mathbf{P}\right) \mathbf{x}_{e}-R^{-1}\left(\mathbf{b}_{n}^{T} \mathbf{P} \mathbf{x}_{e}\right)^{2} .
\end{aligned}
$$

According to Riccati equation (25), (30) will be transformed into

$$
\dot{V}=-\mathbf{x}_{e}^{T} \mathbf{Q} \mathbf{x}_{e}-R^{-1}\left(\mathbf{b}_{n}^{T} \mathbf{P} \mathbf{x}_{e}\right)^{2}
$$

Because $\mathbf{Q}$ is chosen as a positive definite symmetric matrix, there is $\dot{V} \leq 0$. Moreover, when and only when $\mathbf{x}_{e}=0, \dot{V}=0$ holds. Then, the actual state vector $\mathbf{x}$ of system (19) will tend to the desired state vector $\mathbf{x}_{d}$, which reflects that the close-loop control system shown in Figure 3 is stable and possesses the ability of servo tracking. Therefore, the proof of Theorem 3 has been finished.

As a result, the four control parameters in the outer-loop compound controller are designed as follows:

$$
\begin{aligned}
& K_{1}=k_{1}^{*}, \\
& K_{2}=k_{2}^{*}, \\
& K_{3}=B_{n}, \\
& K_{4}=\frac{J_{n}}{B_{n}},
\end{aligned}
$$

where $k_{1}^{*}$ is the first item of vector $\mathbf{k}^{*}$ and $k_{2}^{*}$ is the second item of vector $\mathbf{k}^{*}$.

Thus, for the DC motor system (1), the control law $u$ consists of two parts, and it can be expressed as follows:

$$
u=v-\widehat{d}
$$

So far, the servo controller of DC motor system has been finished, and its control effect will be verified through computer simulation in the next section.

\section{Simulation Results}

In this section, through computer simulation, the control effects of the proposed control scheme are compared with those of PD control scheme and PD + GSMDO control scheme; then we can get the performance advantages of the proposed control scheme.

In simulation, the actual plant parameters are selected as $J=9.0 \times 10^{-3} \mathrm{~N} \cdot \mathrm{s}^{2} /\left(^{\circ}\right), B=6.5 \times 10^{-3} \mathrm{~N} \cdot \mathrm{s} /\left(^{\circ}\right)$, and the nominal parameters are selected as $J_{n}=8.0 \times 10^{-3} \mathrm{~N} \cdot \mathrm{s}^{2} /\left(^{\circ}\right), B_{n}=5.0 \times$ $10^{-3} \mathrm{~N} \cdot \mathrm{s} /\left(^{\circ}\right)$. That is, there exist modeling errors in control 
TABLE 1: The control parameters of control scheme in simulation.

\begin{tabular}{lcccccc}
\hline \multicolumn{2}{c}{ PD controller } & \multicolumn{2}{c}{ Feedforward controller } & \multicolumn{3}{c}{ GSMDO } \\
$K_{1}$ & $K_{2}$ & $K_{3}$ & $K_{4}$ & $k$ & $q$ & $\phi$ \\
\hline 14.1421 & 0.6479 & 0.005 & 1.60 & 5.0 & 2.0 & 0.2 \\
\hline
\end{tabular}

period. Furthermore, we can get the equivalent disturbances caused by the modeling errors, which are expressed by

$$
d_{1}=\left(1-\frac{J}{J_{n}}\right) u-\left(B-\frac{J B_{n}}{J_{n}}\right) \omega .
$$

In addition, considering the impacts of Coulomb friction torque and parameter perturbations in motor control system, the equivalent disturbances caused by them are, respectively, given by

$$
\begin{aligned}
& d_{2}=-0.60 \operatorname{sgn}(\omega), \\
& d_{3}=0.15 \sin (2 \pi t) .
\end{aligned}
$$

Thus, the equivalent disturbance in motor control system is totally expressed by

$$
d=d_{1}+d_{2}+d_{3} .
$$

In simulation, the weighting parameters in quadratic performance index (23) are selected as

$$
\mathbf{Q}=\left[\begin{array}{cc}
1000 & 0 \\
0 & 1
\end{array}\right], \quad R=5
$$

Furthermore, the remainder control parameters of control scheme are shown in Table 1.

In Table 1, parameters $K_{1}, K_{2}, K_{3}$, and $K_{4}$ are illustrated in Figure 3; meanwhile parameters $k, q$, and $\phi$ are shown in (17)-(18). In particular, in practical application, the equivalent disturbance in control system is bounded; however the boundary is unknown in previous. Therefore, the parameters $q$ and $\phi$ can be selected by using the method of trial and error and considering the compromise between the tracking properties of DC motor and the smoothness of control input.

At the same time, taking the actual situation into account, the controller output is limited to $\pm 10 \mathrm{~V}$. In simulation, the curve of position command of DC motor system is shown in Figure 4.

Then, the comparison curves of tracking errors of DC motor systems under the three control schemes are illustrated in Figure 5.

As it can be seen from Figure 5, compared to traditional PD control scheme, the tracking errors of DC motor system with PD + GSMDO control scheme are smaller, which reflects the robust performance of GSMDO against equivalent disturbances. Then, when the compound controller is introduced to PD + GSMDO control scheme, that is, the proposed control scheme is formed, the tracking errors of DC motor system will be smaller again, which reflects the effect of compound controller in improving the tracking accuracy of DC motor system.

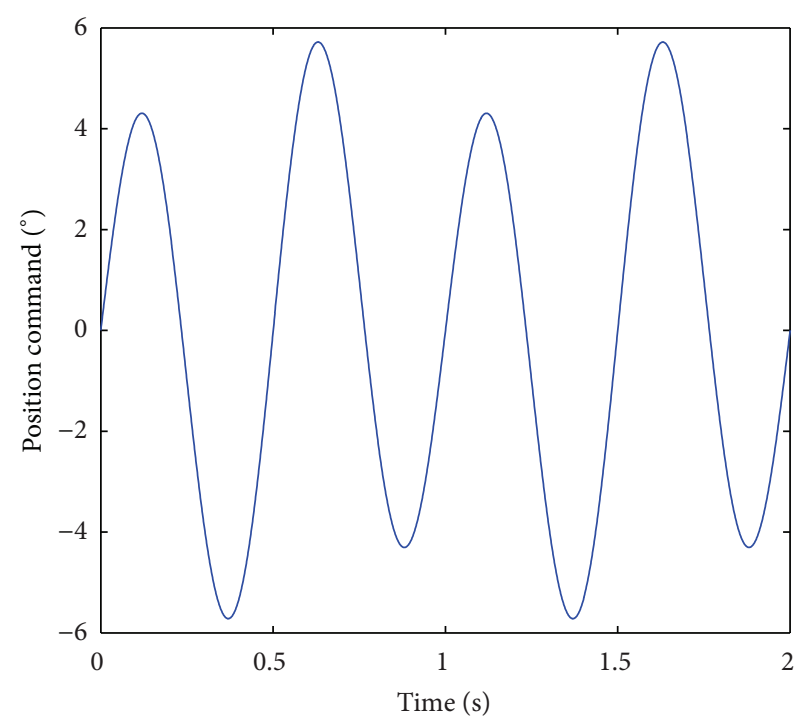

FIGURE 4: The curve of position command of DC motor system.

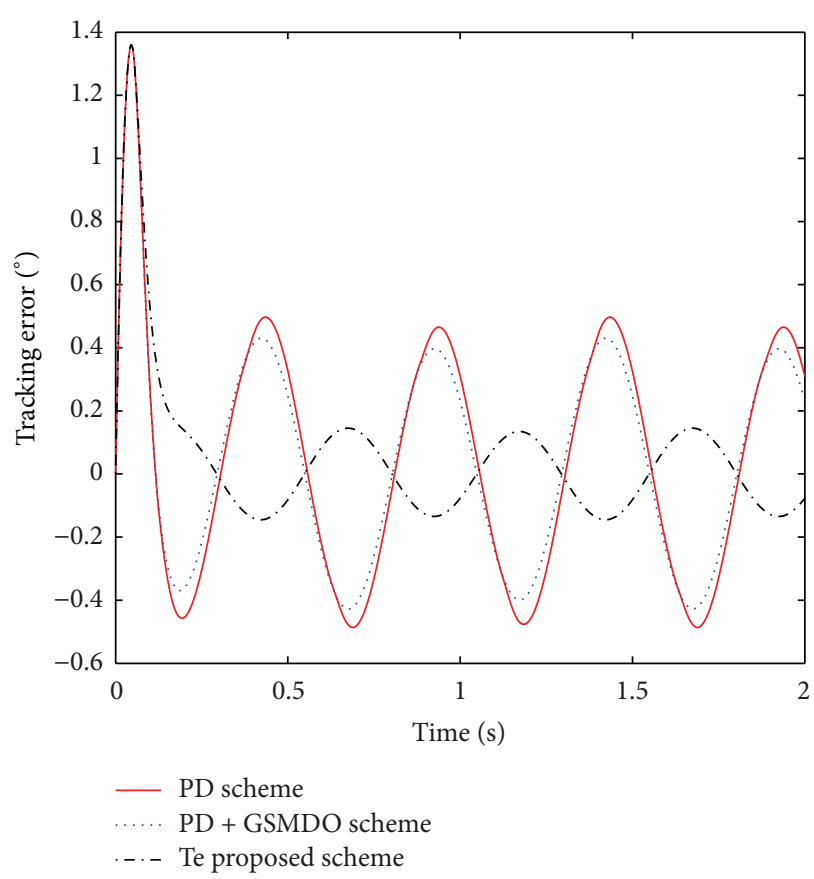

FIGURE 5: The comparison curves of tracking errors of DC motor systems under the three control schemes.

In fact, the robust property of GSMDO results from the accurate estimation on the equivalent disturbances, and then the estimation curves of GSMDO with respect to equivalent disturbances are shown in Figure 6.

As it can be seen from Figure 6, the GSMDO proposed in this paper can reflect the actual level of equivalent disturbances more accurately; therefore the effectiveness of the proposed control scheme can be guaranteed.

Meanwhile, the control voltages calculated by the proposed control scheme are illustrated in Figure 7. 


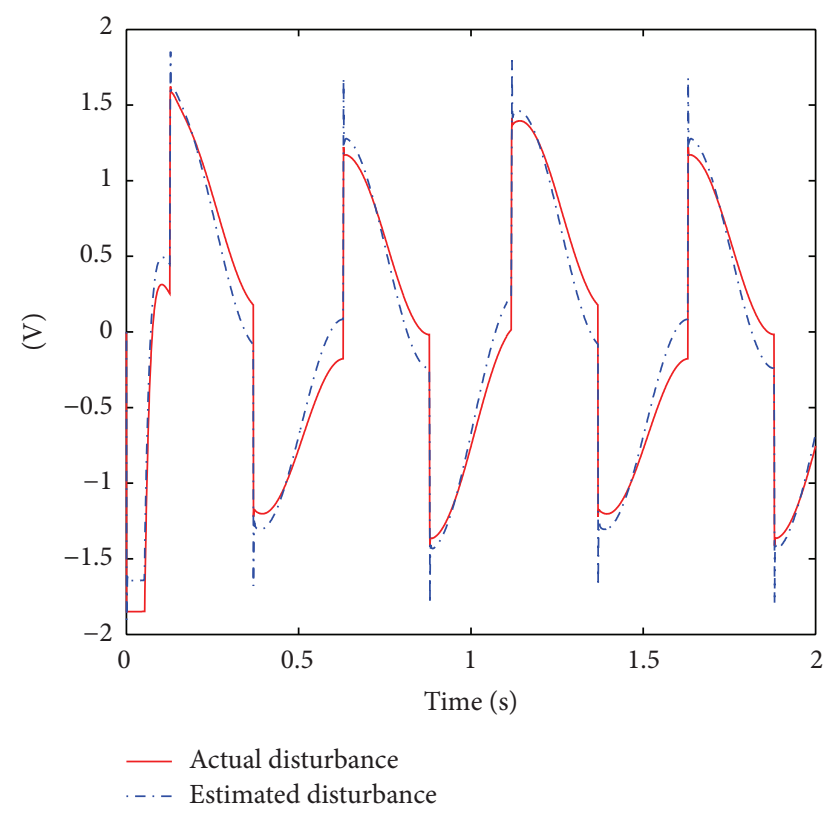

Figure 6: The estimation curves of GSMDO with respect to equivalent disturbances.

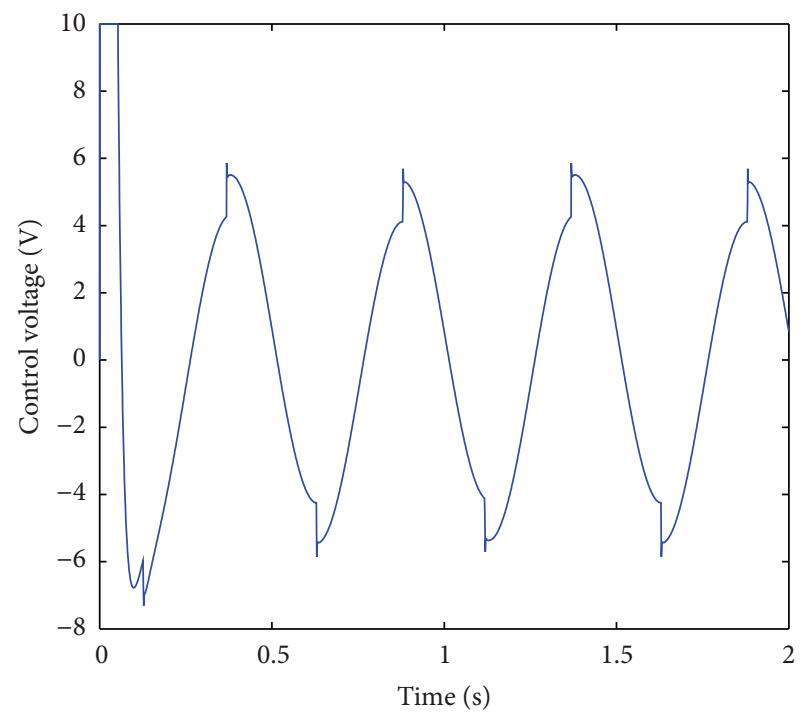

FIgURE 7: The curve of control voltages under the proposed control scheme.

As it can be seen from Figure 7, because of the introduction of the saturation function, there is no significant highfrequency chattering in control quantity, which reduces the impact on the motor mechanical structure and also avoids the instability resulting from the high-frequency chattering.

So far, the computer simulation of the feasibility and the validity of the proposed control scheme has been completed.

\section{Conclusions}

From the simulation results, the two-loop control scheme proposed in this paper, which is constructed by the GSMDO in inner loop and the compound controller in outer loop, can guarantee the better tracking properties of DC motor system. The designed GSMDO can estimate the unknown equivalent disturbances more accurately, which greatly improves the robust property of DC motor control system with respect to system uncertainties, such as modeling errors, parameter variations, and torque disturbances. In addition, the structure of this control scheme is simple; therefore it is easy to be implemented in practical applications. The experimental verification will be needed in future work. Moreover, when the proposed control scheme is applied in actual DC motor system, how to effectively acquire the difference components of control law in a noisy environment should be considered and further solved. As a result, some more excellent estimation algorithms $[15,19,20]$ can be used as references in further work. By using the inner-loop GSMDO, some small unestimated disturbances still exist in motor control system. Though the closed-loop control system with the proposed outer-loop controller possesses a certain control margin against these small disturbances, the improved outerloop controller should be explored in order to get the stronger robustness.

\section{Conflict of Interests}

The authors declare that there is no conflict of interests regarding the publication of this paper.

\section{Acknowledgments}

This work was supported by Research on Pattern Recognition (Grant no. 12ZB342), Oil and Gas Pipeline Detection Technology Research Based on Multisensor Data Fusion (Grant no. 2012XJZ021), Two-Closed-Loop Optimization and Control for Intelligent Well Based on Model Predictive Control (Grant no. 21204139), and Design of Intelligent Well Real Time Optimization and Control System (Grant no. 11ZA023). The projects were supported by Foundation of Sichuan Educational Committee, 2012 Youth Foundation of Southwest Petroleum University, NSFC, and Educational Commission of Sichuan Province of China.

\section{References}

[1] Y. Wu, Y. Liu, and W. Zhang, "A discrete-time chattering free sliding mode control with multirate sampling method for flight simulator," Mathematical Problems in Engineering, vol. 2013, Article ID 865493, 8 pages, 2013.

[2] Y. Ren, Z. Liu, X. Liu, and Y. Zhang, "A chattering free discrete-time global sliding mode controller for optoelectronic tracking system," Mathematical Problems in Engineering, vol. 2013, Article ID 951492, 8 pages, 2013.

[3] X. D. Liu, Y. J. Wu, Y. Deng, and S. Xiao, "A global sliding mode controller for missile electromechanical actuator servo system," Proceedings of the Institution of Mechanical Engineers G: Journal of Aerospace Engineering, 2013.

[4] H. S. Lee and M. Tomizuka, "Robust motion controller design for high-accuracy positioning systems," IEEE Transactions on Industrial Electronics, vol. 43, no. 1, pp. 48-55, 1996. 
[5] B. K. Kim, W. K. Chung, and K. Ohba, "Design and performance tuning of sliding-mode controller for high-speed and highaccuracy positioning systems in disturbance observer framework," IEEE Transactions on Industrial Electronics, vol. 56, no. 10, pp. 3798-3809, 2009.

[6] C. Du, H. Li, C. K. Thum, F. L. Lewis, and Y. Wang, "Simple disturbance observer for disturbance compensation," IET Control Theory \& Applications, vol. 4, no. 9, pp. 1748-1755, 2010.

[7] W. Gao and J. C. Hung, "Variable structure control of nonlinear systems. A new approach," IEEE Transactions on Industrial Electronics, vol. 40, no. 1, pp. 45-55, 1993.

[8] J. Wang, A. B. Rad, and P. T. Chan, "Indirect adaptive fuzzy sliding mode control. I. Fuzzy switching," Fuzzy Sets and Systems, vol. 122, no. 1, pp. 21-30, 2001.

[9] X. Yu and O. Kaynak, "Sliding-mode control with soft computing: a survey," IEEE Transactions on Industrial Electronics, vol. 56, no. 9, pp. 3275-3285, 2009.

[10] S. Tong and H.-X. Li, "Fuzzy adaptive sliding-mode control for MIMO nonlinear systems," IEEE Transactions on Fuzzy Systems, vol. 11, no. 3, pp. 354-360, 2003.

[11] K. C. Veluvolu and Y. C. Soh, "Fault reconstruction and state estimation with sliding mode observers for Lipschitz non-linear systems," IET Control Theory \& Applications, vol. 5, no. 11, pp. 1255-1263, 2011.

[12] Y. Feng, X. H. Yu, and F. L. Han, "High-order terminal slidingmode observer for parameter estimation of a permanentmagnet synchronous motor," IEEE Transactions on Industrial Electronics, vol. 60, no. 10, pp. 4272-4280, 2013.

[13] L. Liu, Z. Han, and W. Li, "Global sliding mode control and application in chaotic systems," Nonlinear Dynamics, vol. 56, no. 1-2, pp. 193-198, 2009.

[14] H. Pang and X. Chen, "Global robust optimal sliding mode control for uncertain affine nonlinear systems," Journal of Systems Engineering and Electronics, vol. 20, no. 4, pp. 838-843, 2009.

[15] Y. Wu, X. Liu, and D. Tian, "Research of compound controller for flight simulator with disturbance observer," Chinese Journal of Aeronautics, vol. 24, no. 5, pp. 613-621, 2011.

[16] D. S. Naidu, Optimal Control Systems, Special Indian Edition, CRC Press, Boca Raton, Fla, USA, 2002.

[17] M. Basin, J. Rodriguez-Gonzalez, and L. Fridman, "Optimal and robust control for linear state-delay systems," Journal of the Franklin Institute, vol. 344, no. 6, pp. 830-845, 2007.

[18] V. Dragan, H. Mukaidani, and P. Shi, "The linear quadratic regulator problem for a class of controlled systems modeled by singularly perturbed Itô differential equations," SIAM Journal on Control and Optimization, vol. 50, no. 1, pp. 448-470, 2012.

[19] X. Wang, S. Yau, and J. Huang, "A study of trackingdifferentiator," in Proceedings of the 39th Decision and Control Conference, pp. 4783-4784, May 2000.

[20] X. Wang, Z. Chen, and G. Yang, "Finite-time-convergent differentiator based on singular perturbation technique," IEEE Transactions on Automatic Control, vol. 52, no. 9, pp. 1731-1737, 2007. 


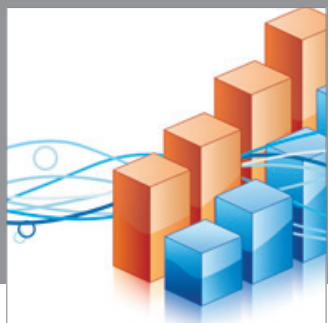

Advances in

Operations Research

mansans

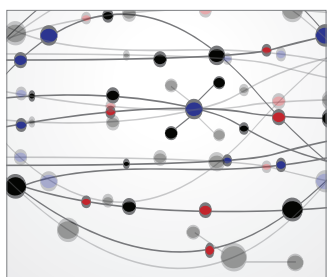

The Scientific World Journal
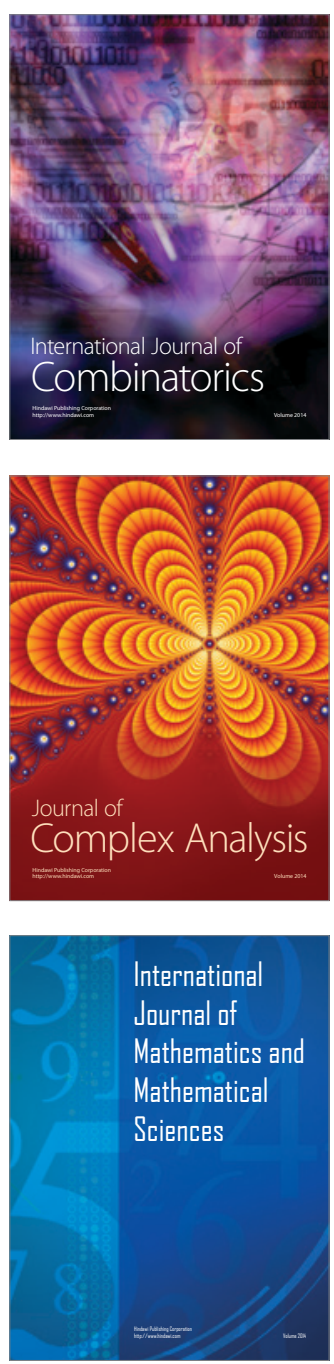
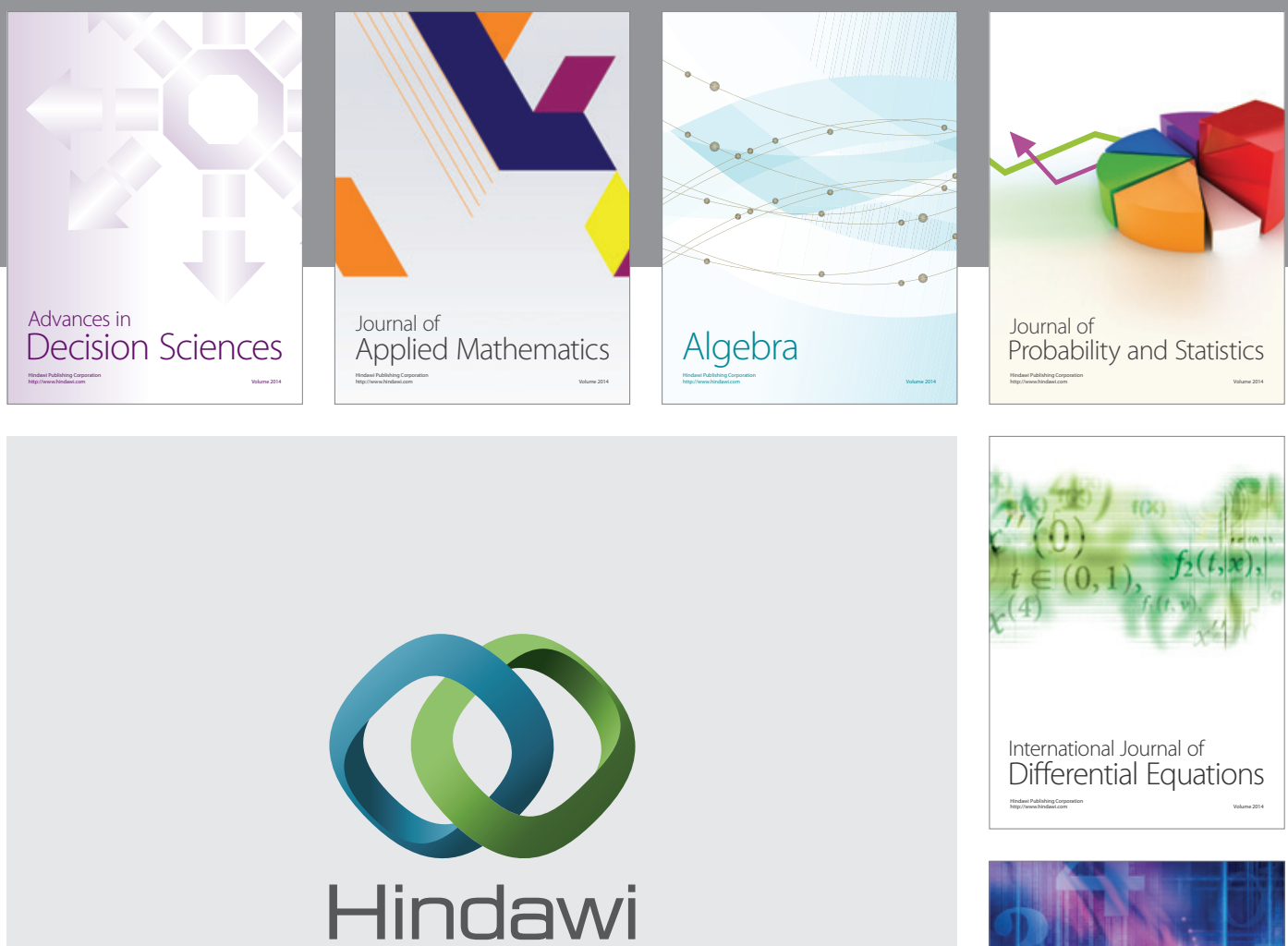

Submit your manuscripts at http://www.hindawi.com
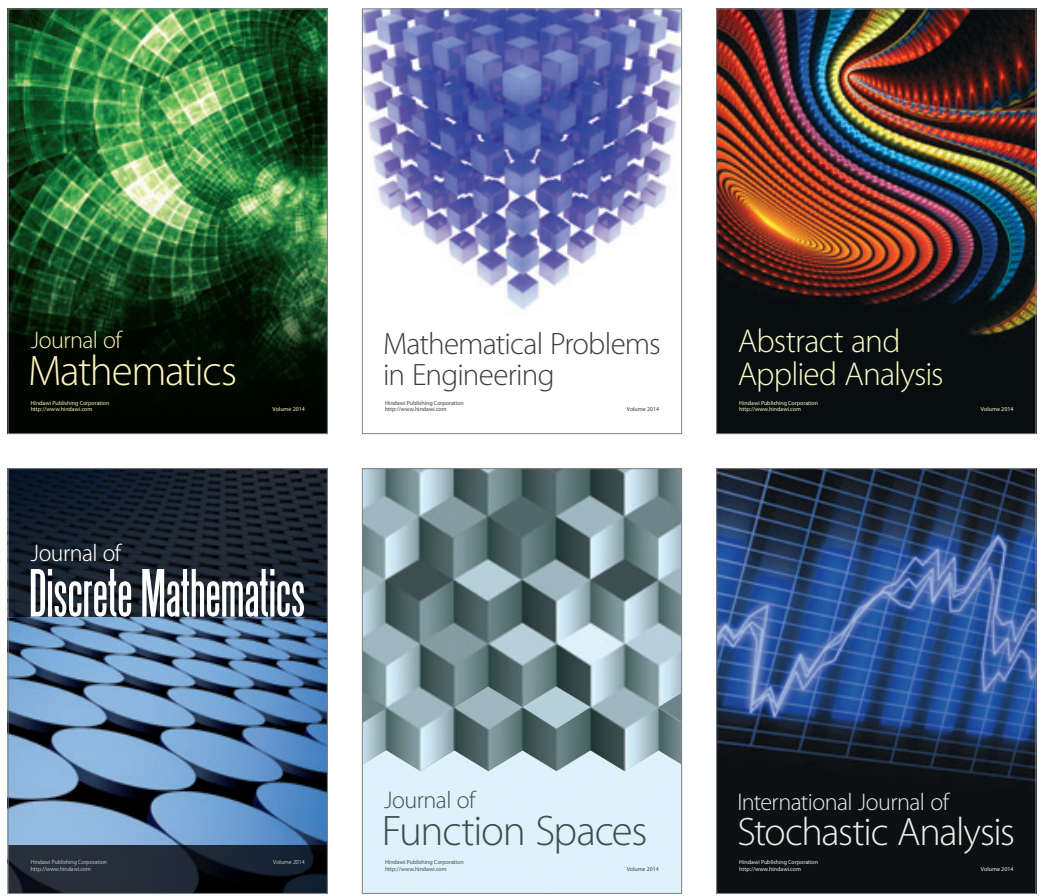

Journal of

Function Spaces

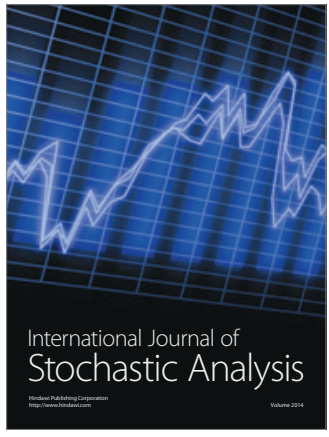

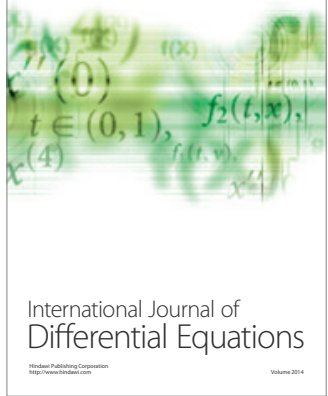
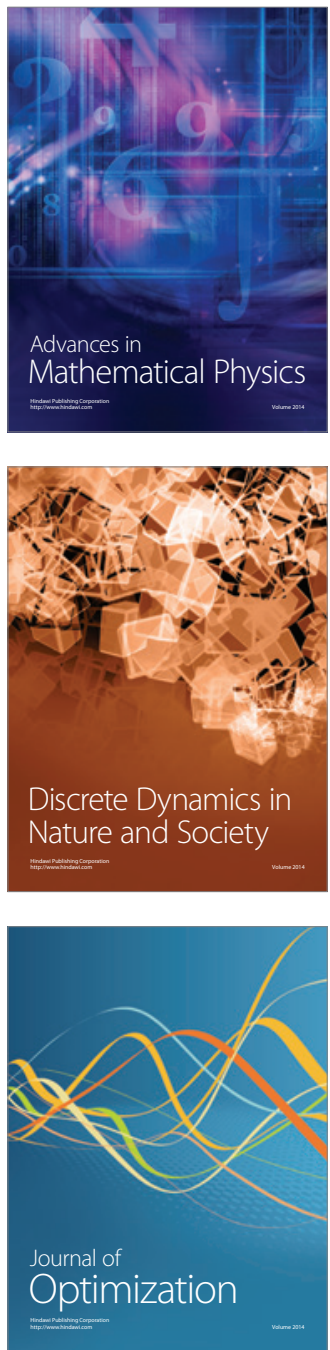